\title{
COMPARISON OF SERUM KIM-1 AND MIOX LEVELS IN PATIENTS THAT UNDERWENT PERCUTANEOUS NEPHROLITHOTOMY AND FLEXIBLE URETERORENOSCOPY
}

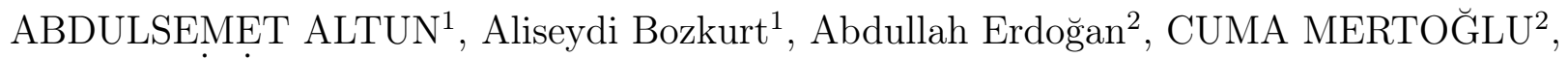 \\ ERKAN HİRIK ${ }^{1}$, Ercüment Keskin ${ }^{2}$, and ABDULLAH TURAN ${ }^{2}$ \\ ${ }^{1}$ Erzincan Binali Yildirim Universitesi \\ ${ }^{2}$ Erzincan Binali Yildirim University
}

April 6, 2021

\begin{abstract}
Aim: Percutaneous nephrolithotomy (PNL) and retrograde intrarenal surgery (RIRS) are common surgical methods in the treatment of kidney stones. Possible effects on kidneys are an important factor in determining the surgical procedure and the surgical method. In our study, kidney injury molecule-1 (KIM-1) and myo-inositol oxygenase (MIOX) were used to compare acute kidney injury in patients that underwent PNL and RIRS. Material and Method:Eighty patients aged 20 to 75 , who underwent PNL or RIRS in our urology clinic between November 2018 and February 2020 were included in the study. In this prospective study, the demographic characteristics, stone size, operation time, preoperative and postoperative hemoglobin and biochemistry values of the patients were recorded. $5 \mathrm{cc}$ blood samples taken from the patients before the operation and at the fourth hour after the operation were centrifuged and kept at $-80{ }^{\circ} \mathrm{C}$, and the KIM-1 and MIOX levels were measured in the biochemistry department. Results: There was no difference between the groups in terms of demographic data; however,the operation time and length of hospital stay were significantly longer in the PNL group. The mean increase in MIOX was $10.583 \pm 9.73 \mathrm{ng} / \mathrm{ml}$ and $7.501 \pm 16.46 \mathrm{ng} / \mathrm{ml}$ in the PNL and RIRS groups, respectively. Although there was a statistically significant increase in both groups, this increase was greater in the PNL group. A significant increase was observed only in the PNL group in the postoperative period $(\mathrm{p}=0.003)$. Discussion and Conclusion:The findings of the study suggest that the PNL procedure causes more damage to the kidneys than RIRS.
\end{abstract}

\begin{abstract}
Aim: Percutaneous nephrolithotomy (PNL) and retrograde intrarenal surgery (RIRS) are common surgical methods in the treatment of kidney stones. Possible effects on kidneys are an important factor in determining the surgical procedure and the surgical method. In our study, kidney injury molecule-1 (KIM-1) and myoinositol oxygenase (MIOX) were used to compare acute kidney injury in patients that underwent PNL and RIRS.

Material and Method: Eighty patients aged 20 to 75, who underwent PNL or RIRS in our urology clinic between November 2018 and February 2020 were included in the study. In this prospective study, the demographic characteristics, stone size, operation time, preoperative and postoperative hemoglobin and biochemistry values of the patients were recorded. $5 \mathrm{cc}$ blood samples taken from the patients before the operation and at the fourth hour after the operation were centrifuged and kept at $-80{ }^{\circ} \mathrm{C}$, and the KIM- 1 and MIOX levels were measured in the biochemistry department.
\end{abstract}


Results: There was no difference between the groups in terms of demographic data; however,the operation time and length of hospital stay were significantly longer in the PNL group. The mean increase in MIOX was $10.583 \pm 9.73 \mathrm{ng} / \mathrm{ml}$ and $7.501 \pm 16.46 \mathrm{ng} / \mathrm{ml}$ in the PNL and RIRS groups, respectively. Although there was a statistically significant increase in both groups, this increase was greater in the PNL group. A significant increase was observed only in the PNL group in the postoperative period $(\mathrm{p}=0.003)$.

Discussion and Conclusion: The findings of the study suggest that the PNL procedure causes more damage to the kidneys than RIRS.

\section{What's already known about this topic?}

PNL and RIRS are common surgical methods.It is known that surgical procedures for kidney stones may have negative effects on kidneys.

\section{What does this article add?}

Serum kim-1 and miox molecules were used for the first time in patients treated with PNL and RIRS. It has been demonstrated with these molecules that PNL does more damage than RIRS.

\section{INTRODUCTION}

Kidney stone disease is one of the most common urinary system diseases. It has a recurrence rate of up to $50 \%$ within five years [1]. Different treatment methods are available in patients with kidney stones according to the size and location of the stone. There are treatment alternatives in kidney stones, such as stone fragmentation with extracorporeal shock wave lithotripsy (ESWL), percutaneous nephrolithotomy (PNL), and retrograde intrarenal surgery (RIRS). PNL is a method of removing stones by first fragmenting them with lithotriptors after percutaneous entry into the kidney. Percutaneous access to the kidney was first reported in 1955 by Goodwin et al., and the first PNL procedure was described by Fernström in 1976 as a treatment option for kidney stones [2; 3]. However, with the introduction of ESWL in the early 1980s, the use of PNL has decreased but it can be an appropriate treatment method, especially for treating large or multiple kidney stones and stones located in the lower calyx [4]. The European Association of Urology (EAU) guidelines also recommend PNL as the first option in the treatment of kidney stones larger than $2 \mathrm{~cm}$ [5]. RIRS is the name given to the process of fragmenting the stone with a laser accessing the kidney through the ureter with a flexible ureterorenoscope. This technique has the advantages of using natural methods and patients requiring shorter hospital stay in the postoperative period. The success of RIRS is related to the size, hardness and location of the stone [6].

When kidney functions are impaired, blood urea and creatinine levels increase, but this may occur hours, even days later. Some markers are used in urine and blood to detect kidney dysfunction earlier. Among the markers that have been shown to be effective in determining kidney damage are some urinary enzymes [7], cystatin C, urine cytokines; myo-inositol oxygenase (MIOX), and kidney damage molecules [e.g., kidney injury molecule-1(KIM-1)] [8; 9].

Although there is no specific blood marker showing kidney damage, KIM-1 is released at high rates in proximal tubular cells after this damage. Although KIM-1 is mostly used as a urine marker for kidney damage, it has been revealed that it can also be detected in the blood to detect kidney injury [10; 11].

Gaut et al. used the Western blot technique and reported that the increase in myo-inositol oxygenase (MIOX), which has been demonstrated to be specific to proximal renal tubules, occurred approximately two days before the increase in the serum creatinine level in kidney damage, and therefore it can be used as a potential early diagnosis sign for acute kidney injury [12].

In this study, we aimed to reveal the possible acute kidney damage caused by these surgical techniques by measuring plasma KIM-1 and MIOX levels at the preoperative and postoperative fourth hour in patients who underwent PNL and RIRS, and to compare these two techniques in terms of these parameters. 


\section{MATERIAL AND METHOD}

This study was conducted at the urology clinic of Erzincan Binali Yıldırım University between November 15, 2018 and February 15, 2020 in accordance with the principles of the Declaration of Helsinki, patient rights regulation and ethical rules, after receiving the ethics committee approval (numbered33216249_604.01.02.E.49628, dated November 9, 2018). Eighty patients scheduled for PNL or RIRS in our urology clinic were included in the study. The patients were informed about the study in advance, and their consent was obtained for participation in the study.

The patients' age, gender, operation time, operation side, stone location, stone size, kidney entry points (in the PNL group), presence of postoperative residual stones, and length of hospital stay were recorded. Three patients were excluded from the study due to solitary kidney, 4 due to renal dysfunction, 1 due to skeletal deformity (scoliosis), and 1 due to ectopic kidney.

Preoperative complete blood count analysis, serum urea analysis, biochemical tests containing creatinine and blood parameters, posterior-anterior chest radiography, ECG, serological tests, and urine culture analysis were performed in all patients. In the postoperative period, complete blood count and urea creatinine values were examined again. In order to reveal the presence of residual stones, non-contrast computed tomography was performed one month after the operation, and stones smaller than $4 \mathrm{~mm}$ were accepted as clinically insignificant residual fragments (CIRFs). Complete stone-free status and CIRFs were considered to indicate operative success. The Clavien classification was used to evaluate the complications that developed.

Venous blood samples were taken preoperatively and four hours after the operation from each patient who underwent surgery in our service and were included in the study. These samples were centrifuged and stored at $-80{ }^{\circ} \mathrm{C}$.

\section{Rirs Technique}

The patients in this group were placed in the lithotomy position, and a semirigid ureteroscope (Karl Storz 9-11F, Tuttlingen, Germany), followed by a9.5-11-F access sheath (Boston Scientific, USA)were inserted with a through a sensor guide (PTFE-Nitinol Guidewire with Hydrophilic Tip, Boston Scientific, Marlborough, USA) under fluoroscopy control. The stone was accessed using a flexible ureteroscope (7,95 F Olympus, USA), and fragmentation was performe dusing Holmium YAG laser with $272-\mu \mathrm{m}$ laser fibers(Quanta System, Litho Holmium, YAG Laser, Turkey).Large fragments were extracted using a 1.7 F retrieval basket (Zerotip, Boston Scientific Corp, Natick, MA, USA).

\section{Pnl Technique}

The patients were placed in the lithotomy position, and following the insertion of a semirigid ureteroscope (Karl Storz 9-11F, Tuttlingen, Germany) and a sensor guide (PTFE-Nitinol Guidewire with Hydrophilic Tip, Boston Scientific, Marlborough, USA) an open-ended ureteral catheter rwas placed under fluoroscopy guidance. After placing the patients in the prone position, retrograde pyelography was performed, and a percutaneous insertion needle was inserted into the appropriate calyx.Under guidance,28-F Amplatz dilatation (Geotec, Ankara, Turkey) was applied to all patients.Stones were reached using a nephroscope (Karl Storz $26 \mathrm{~F}$, Germany), and the stones were fragmented with pneumaticlithotripsy (Elmed, Vibrolith Plus, Turkey). The fragmented pieces were removed from the kidney with stone forceps. A 14-F or 16-F nephrostomy tube was placed in all patients. After fluoroscopy control, antegrade pyelography was performed, and the procedure was terminated.

\section{Statistical analysis}

Data were analyzed using SPSS version 25.0 (SPSS@, IL, USA). The compliance of the data with the normal distribution curve was evaluated using the Shapiro-Wilk test. Continuous variables were compared using Student's t-test and Mann-Whitney Utest, and categorical data were compared using the chi-square test. For the comparison of the preoperative and postoperative data within the groups, the t-test and Wilcoxon test were used in dependent groups. $\mathrm{P}<0.05$ was considered statistically significant. 


\section{RESULTS}

In this prospectively conducted study, no difference was observed between the groups in terms of the mean age, female/male ratio, BMI, presence of DM and stone locations. However, the mean stone dimensions were calculated as $343.65 \pm 236.63 \mathrm{~mm}^{2}$ and $122.48 \pm 51.292 \mathrm{~mm}^{2}$ for the PNL and RIRS groups, respectively, indicating a statistically significant difference (p [?] 0.001) (Table1).

The mean operation time of the patients was $60.25+-22.98$ and $47.50+-20.69$ minutes in the PNL and RIRS groups, respectively, and a statistically significant difference was found between the two groups (p [?] 0.001). The mean length of hospital stay was $4.10+-2.37$ days in the PNL arm and $1.53+-1.61$ days in the RIRS arm, also indicating a statistically significant difference (p [?] 0.001). Stone-free status and presence of CIRFs were accepted as surgical success, and there was no statistically significant difference between the two groups in terms of this outcome. The modified Clavien classification was used for the postoperative complication evaluation. Twenty-six patients (65\%) that had undergone RIRS were evaluated as Clavien 0, $12(30 \%)$ patients as Clavien 1, and $2(5 \%)$ patients as Clavien 2 (infection) (22.5\%). In the PNL group, Clavien 0 was seen in $9(22.5 \%)$ patients, Clavien 2 in $6(22.5 \%)$ (infection in 4, bleeding in 1 , and arteriovenouz fistula in 1), and Clavien 3A in 1 (2.5\%) (double-J stent requirement). There was a statistically significant different between the two groups in terms of the Clavien classification $(\mathrm{p}=0.001)$ (Table1).

Preoperative hemoglobin, urea, creatinine, GFR, MIOX and KIM-1 levels, no significant difference was observed between two groups.In the PNL arm, the post-operative hemoglobin $(12.92+-1.37 \mathrm{~g} / \mathrm{dl})$ was found to be statistically significantly lower than in the RIRS arm (14.98+- $3.30 \mathrm{~g} / \mathrm{dl})$ (p [?] 0.001) (Table 2).

In the intra-group comparisons, there was no statistically significant difference between the preoperative and postoperative values of hemoglobin urea, creatinine, GFR and KIM-1 in patients that had undergone RIRS; however, the mean MIOX value was increased from $17.80+-15.39 \mathrm{ng} / \mathrm{ml}$ preoperatively to 25.30 +- 20.11 $\mathrm{ng} / \mathrm{ml}$ postoperative, and this increase was statistically significant $(\mathrm{p}=0.001)$ (Table 2$)$. Within the PNL group, the hemoglobin value was measured as $14.01+-1.34 \mathrm{mg} / \mathrm{dl}$ preoperatively and $12.92+-1.37 \mathrm{mg} / \mathrm{dl}$ postoperatively, indicating a statistically significant difference (p [?] 0.001). Similarly, in this group, the preoperative and postoperative KIM-1 values were $1.21+-0.44$ and $1.68+-0.58 \mathrm{ng} / \mathrm{ml}$,respectively, and the preoperative and postoperative MIOX values were $15.23+-9.36 \mathrm{ng} / \mathrm{ml}$ and $25.81+-16.13 \mathrm{ng} / \mathrm{ml}$, respectively showing a statistically significant difference (p [?]0.001 for both). No significant difference was observed in terms of the other variables (Table 2). In the intra-group evaluation for the postoperative and preoperative differences, the mean MIOX increase was calculated as $7.501+-16.46 \mathrm{ng} / \mathrm{ml}$ for the RIRS group and 10.583 +- $9.73 \mathrm{ng} / \mathrm{ml}$ in the PNL group, and there a statistically significant difference between the two groups in favor of the latter $(\mathrm{p}=0.001)$. Similarly, while the mean KIM-1 increase was $0.375+-1.13 \mathrm{ng} / \mathrm{ml}$ in the RIRS group, it was $0.471+-0.328 \mathrm{ng} / \mathrm{ml}$ in the PNL group, indicating a statistically significant difference $(\mathrm{p}=0.003)$ (Table 2).

\section{DISCUSSION}

Urinary system stone disease is a pathology that constitutes an important part of urology practice. The treatment of kidney stone disease has changed significantly, especially in the last two decades, with the improvement of tools and reduction in their size due to the development of technology. ESWL, PNL and RIRS are considered as three main treatment methods for the treatment of upper urinary stone disease [13].

PNL is recommended as the preferred minimally invasive method due to its high success rates in patients with a high stone burden $(>20 \mathrm{~mm})$ and complex stones [5]. However, complications, such as hemodynamic impairment, vascular bleeding, and parenchymal bleeding, and acute kidney injury may occur after the operation [14].In recent years, with the technological advances in endourological equipment and increasing surgeon experience, RIRS presents as an alternative to PNL, with lower complication rates [15; 16]. There are publications that consider RIRS to be a very good minimally invasive treatment alternative for intrarenal stones of $<20 \mathrm{~mm}$ and report high stone-free rates even in larger stone sizes [17; 18]. 
During surgical operations for kidney stones, there may be certain damage to the kidneys. Having knowledge of the extent of injury caused by each technique used can guide surgeons in the selection of both patients and surgical techniques. Creatinine is used in daily practice to show kidney injury. However, creatinine is a non-specific marker affected by various factors, including age, gender, muscle density, and liver function. In addition, creatinine levels increase days after kidney damage occurs by which time $50 \%$ or more of renal function is lost [19]. Therefore, new molecules have been used to reveal kidney injury more specifically at an earlier stage. In an experimental animal model investigating one of these molecules, KIM-1, the urine and plasma levels were measured after inducing ischemic kidney injury. It was found that the plasma KIM1significantly increased at the third hour compared to the preoperative levels and remained at a high level until the $96^{\text {th }}$ hour [20]. In the same study, it was shown that the plasma KIM-1 level was correlated with urine the KIM-1 level and significantly higher in individuals with kidney injury compared to healthy individuals. Many studies have evaluated urine KIM-1 levels; however, to the best of our knowledge, there is no study in the literature investigating the role of plasma KIM-1 molecule in revealing possible damage of kidney stone surgery.

In a previous study, the diagnostic sensitivity and specificity of the MIOX molecule released from the proximal tubules of kidneys in showing acute kidney injury were observed as $53.8 \%$ and $81.5 \%$, respectively. Accordingly, it was concluded that MIOX could be used as a marker of acute kidney injury [21]. In addition, when compared to creatinine, MIOX was observed to increase much earlier in case of kidney injury [21].However, a review of the literature shows no study comparing the MIOX molecule in patients that have undergone PNL and RIRS.

In our study, the stone volume of the PNL group was statistically significantly higher than that of the RIRS group ( $\mathrm{p}<0.001)$. This difference was due to the indication of PNL for stones with a larger volume, as recommended in the EAU 2019 urinary system stone disease guidelines. In addition, the operation time was significantly longer in the PNL group $(60.25$ +- $22.98 \mathrm{~min})$ compared to the RIRS group $(47.50+-20.69$ $\min$ ) (p [?] 0.001). We also consider this to be related to the different stone volumes. Similarly, in other studies comparing PNL and RIRS, the stone volume was observed to be higher and the operation time was longer operation times in the former $[13 ; 22]$. However, in a study in which the stone sizes were similar in the two surgery groups, the RIRS duration was found to be longer in lower pole stones [23]. The main reason for the prolongation of this period may be due to the more difficult manipulation of the flexible ureteroscope in lower pole stones. In our study, the length of hospital stay was $4.10+-2.37$ days in the PNL group and $1.53+-1.61$ days in the RIRS group, indicating a statistically significantly longer value for the former ( $\mathrm{p}$ [?] 000.1), which is consistent with the literature [13;22; 23].

In this study, the operative success rates were $92.5 \%$ and $90 \%$ in the PNL and RIRS arms, respectively, and no statistical difference was observed between them. The success rates of the studies in the literature were also similar. However, the postoperative stone-free rates are affected by clinical parameters, such as stone location, obesity, stone size, stone composition and anatomy of the renal calyces. There are studies reporting $77 \%$ to $>90 \%$ success rates in RIRS whereas for PNL, the success rates for lower pole stones of $1-2 \mathrm{~cm}$ and above $2 \mathrm{~cm}$ are given as $92 \%$ and $86 \%$, respectively [25]. While two studies [26;27] showed a higher stone-free rate in the PNL group (92 and 98\%, respectively) than the RIRS group (89 and 95\%, respectively), another study showed a statistically non-significant higher stone-free rate in the RIRS group compared to the PNL group [28]. Lastly, Mehmet et al. determined the stone-free rates as $91.4 \%$ and $87 \%$ in the PNL and RIRS groups, respectively [13].

We used the modified Clavien classification to evaluate the complications and found the rate of complications to be statistically significantly higher in the PNL arm $(\mathrm{p}=0.001)$. Similar to our results, the literature contains studies indicating a higher rate of complications in the PNL arm than in the RIRS arm [23; 28]. While no major complications were observed in our study, $2(5 \%)$ patients in the PNL arm required blood transfusion. Post-operative fever occurred in 7 patients (17.5\%) in the PNL arm and 2 (5\%) patients in the RIRS arm.

In a review reported the most common complications of PNL as extravasation (7.2\%), bleeding requiring 
blood transfusion (11.2 - 17.5\%), and fever (21 - 32.1\%) while rare major complications included septicemia $(0.3-4.7 \%)$, colon injury $(0.2-4.8 \%)$, and pleural injury $(0-3.1 \%)(24)$.

In our study, while there was no difference between the preoperative and postoperative hemoglobin values in the RIRS arm, the postoperative hemoglobin was statistically significant lower compared to the preoperative value in the PNL arm (p [?] 0.001). GyooHwan Jung et al. reported similar data to our results.

In a previous study conducted with RIRS patients, it was observed that MIOX did not increase in the postoperative period [29]. In contrast, the postoperative values of the MIOX molecule statistically significantly increased in our PNL group. The preoperative and postoperative MIOX levels in the RIRS arm were 17.80 +- 15.3 and $25.30+-20.11 \mathrm{ng} / \mathrm{ml}$, respectively, and we found a statistically significant increase in this parameter $(\mathrm{p}=0.001)$. However, the increase in the MIOX values was $10.583+-9.73 \mathrm{ng} / \mathrm{ml}$ in the PNL group versus $7.501+-16.46 \mathrm{ng} / \mathrm{ml}$ in the RIRS arm, indicating a statistically significantly higher increase in the former $(\mathrm{p}=0.001)($ Table 2$)$.

In this study, although there was an increase in the plasma KIM-1 levels in the RIRS arm in the postoperative period, no statistically significant difference was observed. However, the postoperative KIM-1 value in the PNL arm was found to be statistically significantly higher than the preoperative value. Our findings our supported by Balasar et al.[30], who compared PNL, RIRS and mini-PNL, and found an increase in the KIM-1/creatinine values in the PNL and RIRS arms in the postoperative period, and this increase was statistically significantly higher in the PNL group.

We detected a statistically significant increase in both the KIM-1 and MIOX values in the postoperative period among the patients that had undergone PNL. In the RIRS group, this increase was observed only in MIOX. We showed that acute kidney injury occurred in both surgical methods; however, it was greater in PNL.

\section{Limitations}

The limitations of this study include the relatively low number of the patients in our sample and a single blood sample collection in the postoperative period.

\section{CONCLUSION}

In the comparison of the patients that underwent PNL or RIRS, we first demonstrated that acute kidney injury could be detected using the MIOX and plasma KIM-1 molecules, and the increase in these molecules was higher in the PNL arm. The higher increase in the PNL arm suggests that this method causes greater acute kidney injury than RIRS; however, there is a need for further studies with larger case series to support our data.

1. Jungers P, Joly D, Barbey et. al. F: ESRD caused by nephrolithiasis: prevalence, mechanisms, ant prevention. J. Kidney Disease. Vol. 44: 799, 2004

2. Goodwin We, Casey Wc, Woolf W. et al. Percutaneous trocar (needle) nephrostomy in hydronephrosis. J Am Med Assoc. 1955 Mar 12;157:891-4..

3. Fernstrom I, Johansson B. Percutaneous pyelolithotomy. A new extraction technique. Scand J Urol Nephrol. 1976;10(3):257-9

4. Mahmoud Osman, Gunnar Wendt-Nordahl, Katrin Heger et al. Percutaneous nephrolithotomy with ultrasonography-guided renal access: experience from over 300 cases. BJU international, 96(6), 875-878

5. C.Turk (Chair), A.Neisus, A. Petrik et al. EAU Guidelines on Urolithiasis. 2019.

6. Nakada SY, Pearle MS. Ureteropyeloscopy. Conlin MJ; Advanced Endourology. Humana Pres Inc, pp 105-108, 2006

7. Charles G Marguet, W Patrick Springhart, Yeh H Tan et al. (2005). Simultaneous combined use of flexible ureteroscopy and percutaneous nephrolithotomy to reduce the number of access tracts in the 
management of c. BJU international

8. Justin Westhuyzen, Zoltan H Endre, Graham Reece et al. (2003). Measurement of tubular enzymuria facilitates early detection of acute renal impairment in the intensive care unit. Nephrology Dialysis Transplantation, $18(3), 543$.

9. Sushrut S, Waikar J, Bonventre V. Biomarkers for the Diagnosis of Acute Kidney Injury. Nephron Clin Pract 2008;109:c192-c197..

10. Venkata S Sabbisetti, Sushrut S Waikar, Daniel J Antoine et al.(2014). Blood kidney injury molecule-1 is a biomarker of acute and chronic kidney injury and predicts progression to ESRD in type I diabetes. J Am Soc Nephrol . 2014 Oct;25(10):2177-86

11. Kushlinskii NE, Gershtein ES, Naberezhnov DS et al. Kidney Injury Molecule-1 (KIM-1) in Blood Plasma of Patients with Clear-Cell Carcinoma. Bull Exp Biol Med . 2019 Jul;167(3):388-392.

12. Gaut JP, Crimmins DL, Ohlendorf MF et al. Development of an immunoassay for the kidney-specific protein myo-inositol oxygenase, a potential biomarker of acute kidney injury. Clin Chem . 2014 May;60(5):74757.

13. Berkan Resorlu , Ali Unsal, Tevfik Ziypak et al. Comparison of retrograde intrarenal surgery, shockwave lithotripsy, and percutaneous nephrolithotomy for treatment of medium-sized radiolucent renal Stones. World J Urol.

14. Egilmez, Tulga Goren, Mehmet Resit . Predicting surgical outcome of percutaneous nephrolithotomy: validation of the guy's stone score and nephrolithometric nomogram in terms of success and complications. J Clin Anal Med 2015; 6(3): 281-286..

15. Maurice Stephan Michel, Lutz Trojan, Jens Jochen Rassweiler Complications in percutaneous nephrolithotomy. Eur Urol 2007; 51: 899-906.

16. Unsal A, Resorlu B, Atmaca AF et al. Prediction of morbidity and mortality after percutaneous nephrolithotomy by using the charlson comorbidity index. Urology 2012; 79: 55-60.

17. Mariani AJ. Combined electrohydraulic and holmium: YAG laser ureteroscopic nephrolithotripsy of large (greater than $4 \mathrm{~cm}$ ) renal calculi. J Urol 2007; 177: 168-73.

18. El-Anany FG, Hammouda HM, Maghraby HA. Retrograde ureteropyeloscopic holmium: YAG laser lithotripsy for large renal calculi. BJU Int 2001; 88: 850- 3.

19. John A Kellum and Norbert Lameire, Clinical Practice Guideline for Acute Kidney Injury. Kidney Int Suppl 2012; 2(1): 1-138..

20. Venkata S Sabbisetti, Sushrut S Waikar, Daniel J Antoine et al., Blood kidney injury molecule-1 is a biomarker of acute and chronic kidney injury and predicts progression to ESRD in type I diabetes. Journal of the American Society of Nephrology, 25(10), 2177-2186. 2014.

21. Cuma Mertoglu, Murat Gunay, Ali Gurel et al. Myo-Inositol Oxygenase As A Novel Marker In The Diagnosis Of Acute Kidney Injury. J Med Biochem 37: 1-6, 2018.

22. Abdullah Erdoğan, Ercüment Keskin, Abdulsemet Altun, Percutaneous nephrolithotomy versus flexible ureteroscopy in terms of cost-effectiveness in patients with 10-30 mm renal stones. Urologia . 2020 Feb;87(1):41-46.

23. Kursad Zengin, Serhat Tanik, Nihat Karakoyunlu et al., Retrograde Intrarenal Surgery versus Percutaneous Lithotripsy to Treat Renal Stones 2-3cm in Diameter. Biomed Res Int . 2015;2015:914231.

24. Michel MS, Trojan L, Rasweiler JJ. Complications in percutaneous nephrolithotomy. Eur Urol 2007; 51: 899-906. 
25. D M Albala, D G Assimos, R V Clayman et al, . Lower pole I: A prospective randomized trial of extracorporeal shock wave lithotripsy and percutaneous nephrostolithotomy for lower pole nephrolithiasisinitial results. J Urol 2001; 166: 2072-80.

26. Akman T, Binbay M, Ozgor F et al. Comparison of percutaneous nephrolithotomy and retrograde flexible nephrolithotripsy for the management of 2-4 cm stones: a matched-pair. BJU Int . 2012 May;109(9):1384-9.

27. Jeong CW, Jung JW, Cha WH et al. Seoul National University renal stone complexity score for predicting stone-free rate after percutaneous nephrolithotomy. PLoS One. 2013;8:e65888.

28. Gyoo Hwan Jung, Jae Hyun Jung, Tae Sik Ahn et. al., Comparison of retrograde intrarenal surgery versus a single- session percutaneous nephrolithotomy for lower-pole stones with a diameter of 15 to $30 \mathrm{~mm}$ : A propensity score-matching study. Korean J Urol 2015;56:525- 532.

29. Mertoglu C, Bozkurt A, Keskin E et al. Evaluation of the effect of Retrograde Intrarenal Surgery with Myo-Inositol Oxygenase. Pak J Med Sci. 2018;34(1):170-174..

30.Balasar M, Piskin M.M, Topcu C at all. Urinary kidney injury molecule-1 levels in renal stone patients. World J Urol 2016 Sep;34(9):1311-6..

\section{Hosted file}

table.pdf available at https://authorea.com/users/406027/articles/516848-comparison-ofserum-kim-1-and-miox-levels-in-patients-that-underwent-percutaneous-nephrolithotomy-andflexible-ureterorenoscopy 